\title{
Die Strafbarkeit wegen „Conspiracy“ in Nürnberg und ihre Bedeutung für die Gegenwart
}

Hört der amerikanische oder englische Jurist das Wort „conspiracy“ so denkt er wahrscheinlich an kriminelle Machenschaften mehrerer Personen im Bereich Wirtschaftskriminalität, ${ }^{1}$ Drogenschmuggel ${ }^{2}$ und Kartellabsprachen. ${ }^{3}$ Sein deutscher Kollege hingegen kann mit dem Begriff, „Verschwörung“ in aller Regel nicht viel anfangen. Bei der Suche nach einer Definition der Strafbarkeit wegen ,conspiracy“ stößt man bald auf den Satz von Lord Denman aus dem Jahr 1832, wonach die Quintessenz einer strafbaren Verschwörung darin besteht, dass eine Verabredung geschlossen wird, eine unrechtmäßige Tat oder eine rechtmäßige Tat mit unrechtmäßigen Mitteln zu begehen. ${ }^{4}$ Reichlich erhellend ist diese Umschreibung nicht; vielmehr stellen sich weitere Fragen: Was bedeutet ,unlawful“" (,unrechtsmäßig“) in diesem Zusammenhang? Liegt der strafrechtliche Vorwurf letztlich in der geplanten Tat oder allein in der Verabredung? Die Verschwörung wird wegen ihrer mangelnden Präzision zugleich geliebt und gehasst. Während Strafverfolger gerne auf dieses Konzept zurückgreifen, vor allem um Beweisschwierigkeiten aus dem Weg zu gehen, wird es etwa vom US-amerikanischen Jurist und Harvard Professor Francis Sayre (1885-1972) im Jahr 1922 wegen seiner rechtsstaatlichen Mängel scharf kritisiert:

"A doctrine so vague in its outlines and uncertain in its fundamental nature as criminal conspiracy lends no strength or glory to the law; it is a veritable quicksand of shifting opinion and ill-considered thought. "5

Im Statut des Internationalen Militärtribunals findet sich die Verschwörung in Art. 6 neben den Verbrechen der Aggression, den Kriegsverbrechen und Verbrechen gegen die Menschlichkeit. Bislang sind Herkunft und Anwendung der conspiracy in Nürnberg wenig erforscht, ${ }^{6}$ obwohl sie von der Anklageschrift in den Rang eines eigenständigen Verbrechens, formuliert als Anklagepunkt Eins, erhoben wurde. Wenn wir hier der Verschwörungsstrafbarkeit genauer nachgehen und uns fragen, ob sich Spuren davon

1 Smith \& Hogan, Criminal Law, S. 310 ff zum Betrug; so auch schon Justice Jackson, IMT Bd. 8, S. 401/402.

2 Vgl. etwa den Fall DPP v. Nock [1978] 2 All ER 654.

3 Vgl. Berichte der Tagespresse vgl. etwa http://www.timesnews.net/article.php?id=9016425 (besucht am 8.9.2009) oder den Fall Hollinshead [1985] 1 All ER 850 vor dem House of Lords.

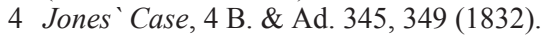

5 Francis B. Sayre, Criminal Conspiracy, Harvard Law Review 35 (1922), 393.

6 In manchen Darstellungen zu den Nürnberger Prozessen findet die Verschwörung kaum Erwähnung; vgl. Klaus Kastner, Von den Siegern zur Rechenschaft gezogen, 2002, S. 134. Auch im Band von Herbert Reginbogin \& Christoph Safferling (Hg.), Die Nürnberger Prozesse, Völkerstrafrecht seit 1945, 2006, findet sich kein eigener Beitrag zur Verschwörung. Eine Ausnahme bildet die jüngst erschienene ausführliche Darstellung von Jonathan Bush, The Prehistory of Corporations and Conspiracy in International Criminal Law: What Nuremberg Really Said, Columbia Law Review 109 (2009), 1094 mit einem Fokus auf die Verantwortlichkeit Industrieller vor allem in den Nachfolgeprozessen. 
auch im modernen Völkerstrafrecht finden lassen, betreten wir - zumal in Deutschland - Neuland.

\section{A. Conspiracy in Nürnberg}

Versuchen wir uns der „,conspiracy“ zu nähern und nehmen wir Nürnberg als Ausgangspunkt, so betrachten wir zunächst das IMT Statut, seinen Wortlaut (I.), seine Interpretation durch Anklagebehörde und Gericht sowie die einzelnen Verurteilungen (II.). Daran anschließend werfen wir einen Blick in die Geschichte des angloamerikanischen Strafrechts (III.) und hoffen, dadurch der Verschwörungsstrafbarkeit auf den Grund zu gehen (IV.).

\section{IMT Statut}

Kriegsminister Stimpson forderte bereits im Sommer 1944, die Verschwörung zum Angriffskrieg in den Mittelpunkt der strafrechtlichen Aufarbeitung zu stellen. ${ }^{7}$ In den schwierigen Beratungen im Sommer 1945 hingegen lag der kleinste gemeinsame Nenner auf der Moskauer Erklärung vom 30.10.1943. Dort standen vor allem die Gräueltaten der Nationalsozialisten in den besetzten Gebieten im Vordergrund und weder der Angriffskrieg noch eine Verschwörungsstrafbarkeit wurde erwogen. ${ }^{8}$ So fand der Begriff „conspiracy“ erst verhältnismäßig spät Einzug in die Entwürfe, da zwischen den vier Delegationen, wie Justice Jackson es ausdrückte, „technische Differenzen“ bestanden. ${ }^{9}$ Im sowjetischen Vorschlag vom 25. 7. 1945 wird von Teilnahme an den Vorbereitungen (preparation) zur Begehung von Aggression, Kriegsverbrechen oder Verbrechen gegen die Menschlichkeit gesprochen. ${ }^{10}$ Der Vorschlag der Delegation der USA vom selben Tag spricht von ,common plan or enterprise“..$^{11}$ Justice Jackson fordert in der anschließenden Aussprache eine „,bessere conspiracy-Vorschrift“ und erwähnt in diesem Zusammenhang ausdrücklich die Verbrechen der Nazis an der eigenen Bevölkerung. ${ }^{12}$ Auch Professor Gros, der Vertreter der französischen Delegation, fordert die Berücksichtigung der Verschwörung, stellt dabei aber nicht auf den Angriffskrieg ab, sondern auf die Verbrechen in den besetzten Gebieten. ${ }^{13}$ Der Streit um diesen Punkt war

7 Henry L. Stimson und McGeorge Bundy, On Active Service in Peace and War, New York, Harper \& Bros., 1947, S. 577 ff. vgl. dazu auch Whitney Harris, Tyrannen vor Gericht, 2008, S. $7 \mathrm{f}$

8 Auf die Moskauer Erklärung wies während der Beratungen vor allem die russische Delegation immer wieder hin. United Nations Information Organisation. London: United Nations, 1945, p. 35 .

9 Vgl. Report of Robert H. Jackson United States Representative to the International Conference on Military Trials, 1945, S. 387 (Justice Jackson.).

10 Ibid. S. 373: „Any person who is proved to have in any capacity whatever directed or participated in the preparation for or carrying out of any of the above-mentioned acts...".

11 Ibid. S. 374.

12 Ibid. S. 380.

13 Ibid. S. 381 f.; das entspricht der allgemeinen französischen Politik hinsichtlich der Nürnberger Prozesse, vgl. Hervé Ascensio, in Herbert Reginbogin \& Christoph Safferling, Die Nürnberger Prozesse: Völkerstrafrecht seit 1945, 2006, S. 36 ff. 
im Übrigen so heftig, dass die Konferenz an diesem Tag zu scheitern drohte. Erst im gemeinsamen Entwurf der britischen und französischen Delegation vom 26./27. Juli 1945 wurde „conspiracy“ zum ersten Mal erwähnt. ${ }^{14}$ Dieser Vorschlag wurde mit geringfügigen Änderungen von Justice Jackson akzeptiert und Art. 6 erhielt seine endgültige Fassung. ${ }^{15}$

Art. 6 IMT letzter Satz: „, Anführer, Organisatoren, Anstifter und Teilnehmer, die am Entwurf oder der Ausführung eines gemeinsamen Planes oder einer Verschwörung zur Begehung eines der vorgenannten Verbrechen teilgenommen haben, sind für alle Handlungen verantwortlich, die von irgendeiner Person in Ausführung eines solchen Planes begangen worden sind. “

Das Anklageteam der USA unter Robert H. Jackson wurde mit der Aufgabe betraut, neben dem Verbrechen des Angriffskrieges (Count Two) auch die Verschwörungsanklage (Count One) vorzubereiten und vor Gericht zu vertreten. ${ }^{16}$ Dabei geht die Anklageschrift davon aus, dass der zitierte letzte Satz von Art. 6 IMT sich auf alle drei vorgenannten Straftaten bezöge. Damit wäre jeder, der sich an einer Verschwörung zur Planung eines Angriffskrieges, an der Planung von Kriegsverbrechen oder Verbrechen gegen die Menschlichkeit beteiligt, wegen conspiracy strafbar. ${ }^{17}$

Verteidiger Seidl setzte seine Interpretation des Textes von Art. 6 IMT dem entgegen. Seine dogmatische Prämisse liegt zunächst darin, festzustellen, dass das Statut autonom interpretiert werden muss und nicht im Lichte einer nationalen Rechtsordnung. Sodann legt er dar, dass systematisch Art. 6 eben nur drei Verbrechenskategorien kennt: Verbrechen gegen den Frieden, Kriegsverbrechen und Verbrechen gegen die Menschlichkeit. ${ }^{18}$ Die Verschwörung wird gerade nicht als eigenständiges Verbrechen erwähnt. ${ }^{19}$

Die Richter des IMT lehnten es in der Tat ab, die conspiracy-Rechtsprechung englischer oder amerikanischer Gerichte zu übernehmen, und fanden eine eigenständige, einschränkende Auslegung als eine Art Zwischenlösung:

„Nach Ansicht des Gerichtshofes fügen diese Worte den bereits aufgezählten Verbrechen kein neues, besonderes Verbrechen hinzu. Die Worte sind dazu bestimmt, die Verantwortlichkeit derjenigen Personen zu bezeichnen, die an einem gemeinsamen Plan teilnehmen. " 20

Nur bezogen auf den Angriffskrieg bezeichnet das Statut die „Verschwörung“ als Verbrechen in Art. 6 a) IMT.

14 Ibid. S. $392 \mathrm{ff}$.

15 S. ibid. S. $394 \mathrm{f}$.

16 Whitney Harris, Tyrannen vor Gericht, S. 28.

17 Eine ähnliche Anklagestrategie wurde im Militärtribunal für den Fernen Osten (Tokio) verfolgt. Die Verschwörung erwies sich dort allerdings als noch diffuser als im NS-Deutschland; vgl. Frederick Mignone, After Nuremberg, Tokyo, Texas Law Review 25 (1947), 475, 482 ff.

18 IMT Bd. 19, $408 \mathrm{f}$.

19 Vgl. auch H. Kraus, Verteidiger des freigesprochenen Hjalmar Schacht: H. Kraus, The Nuremberg Trial of the Major War Criminals: Reflections after Seventeen Years, DePaul Law Review 13 (1963-1964) 233, 239.

20 IMT Bd. 1 S. 253. 
Art. 6a) Verbrechen gegen den Frieden. Nämlich: Planen, Vorbereitung und Einleitung oder Durchführung eines Angriffskrieges oder eines Krieges unter Verletzung internationaler Verträge, Abkommen oder Zusicherungen oder Beteiligungen an einem gemeinsamen Plan oder an einer Verschwörung zur Ausführung einer der vorgenannten Handlungen;

Somit entfällt die Strafbarkeit der Verschwörung zu Kriegsverbrechen oder Verbrechen gegen die Menschlichkeit. ${ }^{21}$ Art. II Nr. 1 a) KRG 10 bestätigt diese Auslegung:

a) Verbrechen gegen den Frieden. Das Unternehmen des Einfalls in andere Länder und des Angriffskrieges unter Verletzung des Völkerrechts und internationaler Verträge einschließlich der folgenden den obigen Tatbestand jedoch nicht erschöpfenden Beispiele: Planung, Vorbereitung, Beginn oder Führung eines Angriffskrieges oder eines Krieges unter Verletzung von internationalen Verträgen, Abkommen oder Zusicherungen; Teilnahme an einem gemeinsamen Plan oder einer Verschwörung zum Zwecke der Ausführung eines der vorstehend aufgeführten Verbrechen.

Bei Verschwörung zur Begehung von Kriegsverbrechen oder Verbrechen gegen die Menschlichkeit wurde in den Nürnberger Folgeprozessen konsequenterweise bereits die sachlichrechtliche Zuständigkeit verneint. ${ }^{22}$

Es scheint daher für das IMT conspiracy keine eigene Verbrechenskategorie zu sein; vielmehr verbirgt sich hinter der Verschwörungsstrafbarkeit eher eine besondere Erscheinungsform des Verbrechens gegen den Frieden, in ihrer dogmatischen Struktur nicht unähnlich der Strafbarkeit des Versuchs. Trotz dieser einschränkenden Herangehensweise bleibt die Verschwörung ein wichtiges Instrument für die Verurteilung der Angeklagten, nicht zuletzt auch deshalb, weil das Verbrechen gegen den Frieden in Nürnberg den wichtigsten Anklagepunkt darstellte. ${ }^{23}$

II. Definition und Anwendung des Verbrechens der Verschwörung durch das IMT in Nürnberg

\section{Definition}

Der Umfang der Verschwörungsstrafbarkeit war in der Anklageschrift denkbar weit gefasst: ${ }^{24}$ jedes Mitglied der NSDAP war im Grunde Teil der Verschwörung, da es

21 Anders noch die Anklageschrift, die „,conspiracy“ auf alle drei Tatbestände des Art. 6 IMT bezog; IMT Bd. 1 S. 30 f. vgl. dazu auch Whitney Harris, Tyranny on Trial, S. 539.; vgl. auch Christoph Safferling, Lernen von Nürnberg, Rechtsgeschichte 14 (2009), S. 148, 161; Robert Herde, Command Responsibility, 2001, S. 172.

22 Vgl. etwa U. S. v. Brands S. 174; U. U. S. v. von Leeb, S. 482 f.; U. S. v. Altstötter, S. 956, 1177.

23 Bruno Simma, The Impact of Nuremberg and Tokyo: Attempts at a Comparison, in N. Ando (ed.), Japan and International Law. Past, Present and Future, 1999, S. 59, 62.

24 Auch in späteren Prozessen versuchte die Anklagebehörde, das Konzept der Verbrechensverschwörung auszuweiten und etwa Industrielle als Mitverschwörer anzuklagen, s. U.S. v. Krupp; diese Auslegung wurde vom Gericht abgelehnt; vgl. H. Kronstein, Columbia Law Review 53 (1953), 139, $141 \mathrm{f}$. 
Kenntnis von den Zielen und Plänen der Partei hatte. ${ }^{25}$ Die Verschwörung ergibt sich aus dem Parteiprogramm der NSDAP aus dem Jahr ihrer Gründung 1921, in dem der Bruch des Versailler Vertrages zum politischen Ziel der Partei erklärt wurde. Die Argumentation der Anklage baute im Wesentlichen auf Hitlers „Mein Kampf“ auf. Im Grunde sollte jedes Parteimitglied, dem dieses Parteiprogramm bekannt war, auch Mitglied der Verschwörung sein. ${ }^{26}$ - Konsequenterweise spricht die Anklageschrift in der Kurzform auch nur noch von Nazi-Conspirators (Nazi-Verschwörer) und nimmt damit terminologisch die Subsumtion unter die Tatbestandsvoraussetzungen bereits vorweg. ${ }^{27} \mathrm{Da}$ es für die Strafbarkeit auch keine Ausführung des gemeinsamen Planes braucht, genügt in der Tat die bloße Mitgliedschaft in der Partei, um die Strafbarkeit zu begründen. ${ }^{28}$ Sir Maxwell-Fyfe führte vor dem IMT aus:

„,Wenn nun Personen übereinkommen, eine ungesetzliche Handlung zu begehen oder eine gesetzliche Handlung mit ungesetzlichen Mitteln auszuführen, so liegt darin von selbst die Begehung einer strafbaren Handlung der Verschwörung. Die Verschwörung entsteht durch das Übereinkommen, nicht erst in den Handlungen zur Ausführung des Übereinkommens. Auf diese Weise beginnt die Verschwörung im Jahre 1921." 29

Diskutiert wurde das Konzept der conspiracy vor allem Ende Februar 1946 häufig auch im Zusammenhang mit der Strafbarkeit der angeklagten Organisationen; eine eindeutige Trennung zwischen den beiden Konzepten wird aus dem Vortrag der Ankläger nicht ersichtlich. ${ }^{30}$ Es ist davon auszugehen, dass beide Modelle - trotz elementarer dogmatischer Unterschiede - für die Ankläger in Nürnberg auf gleicher theoretischer Basis fußen. ${ }^{31}$

Mit dieser weiten Auslegung hatten die Ankläger vor dem IMT allerdings keinen Erfolg. Im Urteil legten die Richter folgende Parameter fest: (1) Der Wortlaut von Art. 6 kennt nur ein „Verschwörungskonzept“", weshalb die Worte „common plan“ und

25 Auch für Jackson war der Grund dafür, dass nicht alle NSDAP-Mitglieder vor Gericht gestellt wurden eher ein tatsächlicher, denn ein rechtlicher: „Nun besteht, wenn man von den aus der Menge der Fälle entstehenden prozessualen Schwierigkeiten einmal absieht, kein Grund, warum nicht jedes Mitglied einer der hier angeklagten Nazi-Organisationen als Teilnehmer der Verschwörung nach Artikel 6 angeklagt und verurteilt werden könnte, selbst wenn das Statut die Organisationen überhaupt nicht erwähnt hätte. Der freiwillige Beitritt war zweifellos eine Handlung, in der sich die Bindung an einen gemeinsamen Plan oder ein gemeinsames Ziel ausdrückte." IMT Bd. 8, 401. Der einzige von ihm akzeptierte Grund für einen Ausschluss der Verantwortung wäre ein Fall, dass jemand sich stets gegen die Ziele der NSDAP ausgesprochen habe und erst in dem Moment in die Partei eintrat, indem Deutschland angegriffen wurde, um sein Vaterland zu verteidigen. IMT Bd. 8, $497 \mathrm{f}$.

26 Vgl. H. Leventhal, S. Harris, J.M. Woosley \& W.F. Farr, The Nuernberg Verdict, Harvard Law Review 60 (1947), 857, $863 \mathrm{f}$.

27 IMT Bd. 1 S. 32.

28 Gerhard Werle, Völkerstrafrecht, 2. Aufl. 2008, Rn. 424.

29 So explizit auf Nachfrage des US-amerikanischen Richters Briddle der britische Chefankläger Sir Maxwell-Fyfe, IMT Bd. 8 S. 243 f.

30 IMT Bd. 8, S. $387 \mathrm{ff}$.

31 Vgl. auch Thomas Vormbaum, Einführung in die moderne Strafrechtsgeschichte, 2009, S. 221. 
„conspiracy“ als identisch angesehen werden müssen. ${ }^{32}$ (2) Eine Mitgliedschaft zur NSDAP allein genügt nicht für die Zurechnung zur Verschwörung. ${ }^{33}$ (3) Eine zeitliche Komponente schränkt die Anwendung ein: „Sie darf vom Entschluß und von der Tat zeitlich nicht zu weit entfernt sein".${ }^{34}$ D.h. es muss vereinbart sein, dass der Plan demnächst in die Tat umgesetzt wird. (4) Substantiell ist zu prüfen, ob es tatsächlich einen solchen konkreten Plan gab, einen Krieg zu beginnen, und wer die Teilnehmer an diesem konkreten Plan sind. „Der Gerichtshof muß untersuchen, ob ein konkreter Plan zur Kriegsführung bestand, und bestimmen, wer an diesem konkreten Plan teilgenommen hat". 35

Es ist nicht einfach, hieraus die objektiven und subjektiven Tatbestandsmerkmale nach deutscher Strafrechtsdogmatik - bzw. actus reus und mens rea - nach angloamerikanischer Terminologie - herauszuschälen. Zunächst muss ein Zusammenschluss (,combination“) mehrerer Personen objektiv vorliegen. ${ }^{36}$ Dieser muss objektiv bezogen sein auf das Führen eines Angriffskriegs. Der Angeklagte muss Teil dieses Zusammenschlusses sein und vor allem subjektiv Wissen über diese Verabredung haben. Es ergibt sich also ein verhältnismäßig klares Bild von den Voraussetzungen, die immerhin über das Wissenselement an den strafrechtlichen Schuldgrundsatz rückgekoppelt sind. ${ }^{37}$

\section{Verurteilungen und Freisprüche}

Betrachten wir das Urteil, so stellen wir fest, dass acht der 21 Angeklagten wegen Anklagepunkt 1 verurteilt wurden. ${ }^{38}$ Bei Frank, Reichsminister ohne Portfolio, Präsident der Akademie für deutsches Recht, wurden die Voraussetzungen der Verschwörung zum Angriffskrieg für nicht gegeben angesehen, und eine Strafbarkeit mit den Worten abgelehnt, Frank sei nicht „,eng genug verbunden“ mit dem Plan Angriffskriege zu führen. ${ }^{39}$ Hinsichtlich Julius Streicher wurde eine Strafbarkeit wegen Verschwörung zum Angriffskrieg ebenfalls abgelehnt, da er nie Teil des Kernbereichs um Adolf Hitler war, an keiner der relevanten Konferenzen zur Kriegsplanung teilgenommen hat und damit nicht nachgewiesen werden konnte, dass er Kenntnis von den Plänen zum Angriffskrieg hatte. ${ }^{40}$

32 IMT Bd. 1 S. 250.

33 IMT Bd. 1 S. 251. Der Verteidiger Servatius hatte auf diesen Unterschied zwischen Verschwörung, wo es einer Verabredung aller Beteiligter bedarf, und Mitgliedschaft, wo nicht einmal Kenntnis einer Verabredung erforderlich ist, deutlich hingewiesen; IMT Bd. 8, 527. Im deutschen Recht wäre eine solch rein objektive Strafbarkeit für die Mitgliedschaft nicht strafbar.

34 IMT Bd. 1 S. 251.

35 IMT Bd. 1 S. 251; Whitney Harris, Tyrannen vor Gericht, S. 544.

36 Vgl. auch Markus Dubber, Einführung in das US-amerikanische Strafrecht, S. 128; Smith \& Hogan, Criminal Law, S. $318 \mathrm{f}$.

37 Vgl. auch Christoph Burchard, The Nuremberg Trial and it's Impact on Germany, Journal of International Criminal Justice 4 (2006), 800, 807.

38 Das waren: Göring, von Ribbentrop, Keitel, Rosenberg, Jodl, zum Tode, Hess und Raeder zu lebenslanger Haft, sowie von Neurath zu 15 Jahren.

39 IMT Bd. 1 S. 334.

40 IMT Bd. 1 S. 340. 
Daraus wird ersichtlich, dass die Kenntnis ein entscheidender Faktor der Zurechnung ist. ${ }^{41}$ Die Nähe zu Hitler, wie sie Frank zweifellos hatte, mag zwar indiziellen Charakter haben, ist aber kein hinreichender Zurechnungsgrund. Ebenso wenig genügt eine ideologische Verblendung, wie sie bei Streicher zu Tage trat.

Auch Dönitz, der spätere Nachfolger Hitlers, wurde vom Vorwurf der Verschwörung freigesprochen, da er lediglich als Marineoffizier tätig war und (jedenfalls zunächst) an den Planungen eines Angriffskrieges keinen Anteil hatte. ${ }^{42}$ Damit wurde auch eine etwaige rückwärtige Strafbarkeit bei sukzessiver Kenntnis von der Verschwörung abgelehnt. Die anderen angeklagten Militärs, wie Raeder, Keitel und Jodl wurden deshalb der Verschwörung zum Angriffskrieg für schuldig befunden, weil ihnen die NS-Politik der Schaffung neuen Lebensraums im Osten bekannt war. Das reichte für die Einbeziehung in die Verschwörung aus. ${ }^{43}$

Wenn der Verteidiger Martin Löffler darauf hinweist, dass das Verschwörungs- oder Verabredungskonzept im Falle des nationalsozialistischen Plans, Europa zu erobern, nicht passt, weil der gemeinsame Plan gerade ein geheimer Plan sein muss, was bei mehreren Millionen Verschwörern kraft Natur der Sache nicht der Fall sein kann, ${ }^{44}$ verwechselt er den Anknüpfungspunkt: Bei internationalen Verbrechen ist der Bezugspunkt die Weltgemeinschaft und nicht die nationale Gesellschaft. Quantitäten verschieben sich demnach.

\section{Herkunft der Verschwörungsstrafbarkeit}

Um den Umgang des Tribunals mit der Verschwörungsstrafbarkeit bewerten zu können, müssen wir einen Blick in die Entwicklungsgeschichte dieses strafrechtlichen Instituts werfen. Als Anknüpfungspunkt diente der Anklagebehörde das angloamerikanische Konzept der Verbrechensverschwörung. So lesen wir bei Whitney Harris:

„Das Konzept der verbrecherischen Verschwörung ging nicht über die Doktrin der Verschwörungsverbrechen hinaus, wie sie im nationalen Strafrecht des anglo-amerikanischen Rechtssystems anerkannt ist. Das Urteil des Gerichtshofes befand lediglich, dass nach dem Völkerrecht Männer, die bei geheimen Treffen den Plan gefasst hatten, eine befreundete Nation zu stürzen, worauf in zeitlicher Nähe Aktivitäten zur Umsetzung dieses Plans folgten, genauso einer verbrecherischen Verschwörung schuldig sind, wie nach innerstaatlichem Recht Männer schuldig sind, die sich unter ähnlichen Umständen verschwören, eine Bank zu überfallen oder ein Kind zu entführen. Dieses Ergebnis wich in keiner Weise von etablierten Rechtsprinzipien ab, wenn auch sicherlich die Anklage für sich das Recht beansprucht hatte, nach dem Statut eine weitaus umfangreichere Verschwörung zu beweisen. "45

41 Darauf hatte auch Martin Löffler, Verteidiger der SA, deutlich hingewiesen, IMT Bd. 8, S. 459.

42 Whitney Harris, Tyrannen vor Gericht, S. 537.

43 Vgl. Christoph Safferling, Lernen von Nürnberg, Rechtsgeschichte 14 (2009), S. 148, $161 \mathrm{f}$.

44 Verteidiger für die SA, IMT Bd. 8, S. 453.

45 Whitney Harris, Tyrannen vor Gericht, S. 539. 
Ebenso Sir Maxwell-Fyfe vor dem IMT:

„In England ist die Verschwörung eine strafbare Handlung nach gemeinem Recht. Es gibt auch gewisse rechtlich festgelegte strafbare Handlungen, aber die strafbare Handlung der Verschwörung bestimmt sich nach gemeinem Recht. "46

Interessanterweise hat die „Verschwörung“ eine sehr spezielle Geschichte. ${ }^{47}$ Die Ursprünge gehen weder auf normannisches noch auf frühes common law zurück, sondern finden sich in frühen Gesetzen König Edwards I von 1285 und $1330 .{ }^{48}$ Der Anlass war die Verhinderung von Missbrauch im Strafprozess, speziell zur Eindämmung falscher Verdächtigungen. Dabei hatte sich offenbar im 13. Jh. folgende Praxis herausgebildet: Stellte sich die Anklage als falsch heraus, musste der Ankläger Strafe zahlen. Um dies zu umgehen, wurden strafunmündige Kinder unter zwölf Jahren vorgeschickt, die falschen Verdächtigungen vorzubringen und dadurch den Gegner in Misskredit zu bringen. Ebenso konnte man durch „öffentliche“ Grand Jury-Anklagen ein persönliches Risiko minimieren. Dieses Vorgehen sollte durch die Verschwörung unterbunden werden. Es handelte sich demnach um eine Maßnahme zum Schutz des öffentlichen Strafverfahrens. Außerdem war dieses ,crime of conspiracy for procuring false indictments“ erst dann vollendet, wenn nach Abschluss des Verfahrens feststand, dass die Verdächtigungen falsch waren. ${ }^{49}$ Erst im Jahre 1611 stellte die berüchtigte „Star Chamber“ im sog. Poulterers' Case $^{50}$ den Analogschluss auf, dass die Strafbarkeit wegen conspiracy auch besteht, wenn es zu keinem Verfahren gegen die Verleumdeten kam. Damit stand fest, dass nicht die falsche Verdächtigung selbst den Strafgrund darstellte, sondern die Verschwörung, d.h. das gemeinsame Planen. ${ }^{51}$ Im Verlauf des 17. Jahrhunderts wurde die weitere Verselbständigung der Verschwörung dann ausgebaut. Es folgte die Loslösung von der Verleumdung als „Grunddelikt“" und die Einbeziehung anderer beliebiger Delikte. Nach Sayre zeigt sich darin eine Entformalisierungstendenz hin zu einer stärkeren Einbeziehung moralischer Werte durch die Gerichte. ${ }^{52}$ Verschwörung zu „unmoralischem Verhalten“" wurde von der King's Bench ebenso sanktioniert, wie die Verabredung zu einem Verbrechen.

Hawkins Pleas of the Crown (1716) führte zum Verbrechen der Verschwörung aus:

"There can be no doubt, but that all confederacies whatsoever, wrongfully to prejudice a third person, are highly criminal at common law. "53

Daraus ergab sich die Überzeugung, dass „,bare conspiracy to do a lawful act to an unlawful end is a crime, though no act be done in consequence thereof." 54 Das endete schließlich 100 Jahre später in der Formel von Lord Denman: a conspiracy indictment

46 IMT Bd. 8, 245.

47 George Fletcher, Rethinking Criminal Law, 2000, S. 646 f.

48 Vgl. dazu Francis B. Sayre, Criminal Conspiracy, Harvard Law Review 35 (1922), 392, 394-396.

49 Francis B. Sayre, Criminal Conspiracy, Harvard Law Review 35 (1922), 392, 397.

509 Coke 55 b.

51 Francis B. Sayre, Criminal Conspiracy, Harvard Law Review 35 (1922), 392, 399.

52 Francis B. Sayre, Criminal Conspiracy, Harvard Law Review 35 (1922), 392, $400 \mathrm{ff}$.

53 Hakins, Pleas of the Crown (1716), 6 ed., bk. 1, c. 72, § 2, S. 348.

54 Rex v. Edwards 8 Mod. 320 (1724). 
must "charge a conspiracy either to do an unlawful act or a lawful act by unlawful means." 55 Diese Formel zitiert auch Justice Jackson als normative Grundlage seiner Verschwörungsanklage. ${ }^{56}$

Die tatsächlichen Auswirkungen dieser denkbar weiten und dehnbaren Formel - „unlawful"“ wurde auch als unmoralisches Verhalten interpretiert ${ }^{57}$ - zeigten sich vor allem im Arbeitskampf der frühen Industrialisierung. ${ }^{58}$ In verschiedenen Fällen sowohl in England wie in den USA wurden Arbeiter wegen „Verschwörung zum Streik“verurteilt, mit dem Argument, dass die Verabredung zum Arbeitskampf ungebührlichen Druck auf den Arbeitgeber ausübte. ${ }^{59}$ Auf diese Art und Weise wurde im Handumdrehen die Arbeiterklasse kriminalisiert. ${ }^{60}$

Man ging teilweise auch so weit, dass dem einzelnen Mitglied der Verschwörung Taten ,zugerechnet“" wurden, die vor seiner Entscheidung, unter die Verschwörer zu gehen, begangen wurden. In diesem Sinne äußert sich auch Sir Maxwell-Fyfe, der diese dem Schuldgrundsatz krass widersprechende Folgerung in Nürnberg vorsichtig in Zweifel zog:

„Ich möchte ein Beispiel anführen, das zwar reichlich phantastisch ist, die Problemstellung aber doch aufzeigt. Nehmen wir eine Verschwörung von Straßenarbeitern, Eisenbahnzüge zum Entgleisen zu bringen, an. Einige Arbeiter verabreden sich im Dezember, am 1. Januar einen Zug zum Entgleisen zu bringen und einen weiteren am 1. Februar. Zwischen dem 1. Januar und dem 1. Februar tritt ein weiterer Eisenbahnarbeiter dieser Verschwörung bei. In diesem Fall ist es meiner Meinung nach zweifelhaft, ob dieser Eisenbahnarbeiter für einen Mord verantwortlich gemacht werden kann, der begangen wurde, als der Zug am 1. Januar zum Entgleisen gebracht wurde. "61

Die Gruppenhaftung wurde im Jahr 1946 in der bekannten Supreme Court-Entscheidung Pinkerton v. U.S. ausgeweitet, indem jeder Verschwörer als Teilnehmer jeder Tat, die ein Mitverschwörer ,zur Förderung“ der Verschwörung begeht, angesehen wird, unab-

55 Jones 'Case, 4 B. \& Ad. 345, 349 (1832); in einem späteren Fall hat Lord Denman diese Formel selbst aber widerrufen: Reg. v. Peck, 9 A. \& E. 686, 690 (1839) Lord Denman antwortete dem Anwalt, der ihm seine eigene Formel vorhielt: "I do not think the antithesis very correct.".

56 S.o. IMT Bd. 8, 402.

57 Smith \& Hogan, Criminal Law, S. 295.

58 See G. Robertson, Whose Conspiracy? NCCL 1974, S. 13-18.

59 Etwa in England: Rex v. Bykerdike 1 Mood. \& Rob. 179 (1832) oder in den USA: State v. Donaldson 32 N. J. L. 151 (1867).

60 Weitere Beispiele bei Francis B. Sayre, Criminal Conspiracy, Harvard Law Review 35 (1922), 392, 400-409.

61 IMT Bd. 8, 245; in Details anders hier der US-amerikanische Recht, wie es von Robert Jackson später erläutert wird, IMT Bd. 8, $402 \mathrm{ff} .:$ „Es ist für die Verantwortlichkeit nicht erforderlich, dass jemand zur gleichen Zeit wie andere Täter oder zur Zeit der Begehung verbrecherischer Handlungen Mitglied einer Verschwörung ist. Wenn jemand Teilnehmer einer Verschwörung wird, macht er sich alles Vorangegangene zu eigen und heißt es gut; er bleibt so lange verantwortlich, bis er aus der Verschwörung unter Benachrichtigung seiner Mitverschwörer ausscheidet.". 
hängig davon, ob er von dieser Tat Kenntnis hatte. ${ }^{62}$ Als mitangeklagter Verschwörer hat man kaum eine Chance sich zu verteidigen. ${ }^{63}$

Francis Sayre findet im Jahr 1922 denkbar harte Worte, um das Rechtsinstitut der conspiracy zu geißeln:

„, Such a doctrine grew out of an historical mistake, and has no real basis in our law. It is logically unsound and indefensible. Moreover, it is dangerous. It tends to rob the law of predictability, and to make justice depend too often upon the chance prejudices and convictions of individual judges. "64

Und auch bei Justice Jackson lesen wir im Jahr 1949 im Fall Krulewitch v. U.S.:

„, The modern crime of conspiracy is so vague that it almost defies definition. "65

Er sah allerdings im Fall der deutschen Kriegsverbrecher weniger Probleme in der Anwendung. Der Grund dafür liegt wahrscheinlich in dem politischen Charakter, dem er der Verschwörung grundsätzlich zumaß:

„, The crime comes down to us wrapped in vague but unpleasant connotations. It sounds historical undertones of treachery, secret plotting and violence on a scale that menaces social stability and the security of the state itself. 'Privy conspiracy' ranks with sedition and rebellion in the Litany's prayer for deliverance. Conspiratorial movements do indeed lie back of the political assassination, the coup d'etat, the putsch, the revolution, and seizures of power in modern times, as they have in all history".

Seit Beginn des 20. Jahrhunderts mehren sich die Stimmen, dass eine Verabredung zwischen mehreren Personen allein keinen Strafgrund darstellen kann und die Verabredung sich auf ein Verbrechen beziehen muss, um Kriminalunrecht darzustellen. ${ }^{66}$ Bis heute hat sich das allerdings in der Rechtsprechung nicht durchgesetzt. ${ }^{67}$ Auch der Model Penal Code in den USA behält die Verschwörungsstrafbarkeit bei, versucht die

62 Pinkerton v. United States, 328 U.S. 640, 648. In diesem Fall ging es um Verstöße gegen das Einkommensteuergesetz (Internal Revenue Code). Justice Jackson war an dieser Entscheidung nicht beteiligt.

63 Das streicht auch Justice Jackson heraus in seiner concurring opinion im Fall Krulewitch v. United States, 336 U.S. 440, 454: „A co-defendant in a conspiracy trial occupies an uneasy seat. There generally will be evidence of wrongdoing by somebody. It is difficult for the individual to make his own case stand on its own merits in the minds of jurors who are ready to believe that birds of a feather are flocked together. If he is silent, he is taken to admit it and if, as often happens, co-defendants can be prodded into accusing or contradicting each other, they convict each other".

64 Francis B. Sayre, Criminal Conspiracy, Harvard Law Review 35 (1922), 392, 427.

65 Justice Jackson concurring opinion im Fall Krulewitch v. United States, 336 U.S. 440, 446. In dem Fall ging es um die Verabredung zwischen einem Mann und einer Frau, eine weitere Frau zur Prostitution angestiftet zu haben.

66 Chief Justice Parker in National Protective Association v. Cumming 170 N. Y. 315, 63 N. E. 369 (1902); s. auch Lindsay and Company v. Montana Federation of Labor 37 Mont. 264, 96 Pac. 127.

67 Vgl. in England: Smith \& Hogan, Criminal Law, S. 296 sowie die Kritik bei Nicola Lacey, Celia Wells \& Oliver Quick, Reconstructing Criminal Law, S. 298-309. 
schlimmsten Auswüchse einzudämmen und beschränkt die Strafbarkeit auf die Verabredung einer Straftat. ${ }^{68}$

Zusammenfassend lässt sich feststellen, dass die Verschwörung ein disparates, ja diffuses Konzept darstellt. Bis heute dient es dazu, Gruppen zu kriminalisieren und Individuen auf möglichst breiter Basis zur Verantwortung zu ziehen. Es ist weder ein konkretes Zurechnungskonzept zu erkennen, noch ist die Rechtsfolge klar.

\section{Strafgrund}

Mängel in der dogmatischen Ausgestaltung könnten evtl. durch einen klar umrissenen Strafzweck ausgeglichen werden. Aber auch hinsichtlich des Strafgrundes lassen sich mehrere Ansätze erkennen. Der ursprüngliche Zweck, der Schutz der Rechtspflege, ist mittlerweile sicher überholt. Die moderne conspiracy bedeutet Vorfeldkriminalisierung. Damit einher geht die Möglichkeit, mit strafprozessualen Mitteln weit vor Eintritt der konkreten Gefährdung gegen die möglichen Täter vorzugehen. Gegebenenfalls helfen diese Ermittlungsmethoden, die Durchführung des Planes zu verhindern. ${ }^{69}$ Aber auch in Fällen, in denen das Verbrechen tatsächlich begangen wurde, was für die Strafbarkeit nicht erforderlich ist, kann die conspiracy helfen, die Beteiligten zu verbinden. ${ }^{70} \mathrm{Wäh}$ rend die Beweisbarkeit von Mittäterschaft oder Beihilfe im Rechtssinne möglicherweise Schwierigkeiten bereitet, kann eine Verabredung leichter bewiesen werden. ${ }^{71}$

Zur Begründung der Vorfeldkriminalisierung werden zwei Standpunkte vertreten: (1) Eine objektive Theorie sieht den hohen Grad an Gefährlichkeit durch die gemeinsame Planung eines schweren Verbrechens; ${ }^{72}$ (2) eine subjektive Theorie beschreibt den Zweck der conspiracy als Identifizierung ,gewissenloser Menschen“. ${ }^{73}$ Klar zu Tage tritt hier ein gesinnungsstrafrechtlicher Einschlag, der dem modernen Tatstrafrecht fremd ist. Auch Jackson scheint in diesem Punkt eher Objektivist zu sein, wenn er in Nürnberg das Lehrbuch von Miller zur Begrünung der conspiracy zitiert:

„, Der Grund dafür, daß ein Tatbestand für strafbar erklärt wird, (...) liegt darin, daß eine Vereinigung von Personen, die sich zur Begehung einer im Zweck oder in den Mitteln unrechten Handlung zusammenschließt, um so vieles gefährlicher ist; denn ihre Macht, Unrecht zu tun, ist stärker, und es ist schwieriger, sich gegen eine Gruppe

68 Vgl. Markus Dubber, Einführung in das US amerikanische Strafrecht, S. 131-133.

69 Antonio Cassese, International Criminal Law, 2. Aufl. 2008, S. 227.

70 Hinsichtlich der gleichzeitigen Strafbarkeit nach Vollendung und Verschwörung bestehen einige Unsicherheiten. Nach dem MPC ist jedenfalls eine insoweit „doppelte“ Verurteilung möglich; vgl. Markus Dubber, Einführung in das US-amerikanische Strafrecht, S. 134.

71 Vgl. dazu auch Robert Cryer, Prosecuting International Crimes, 2005, S. 309-311.

72 Francis B. Sayre, Criminal Conspiracy, Harvard Law Review 35 (1922), 392, 420 findet hier allerdings ein gewichtiges Gegenargument mit Blick auf Großunternehmen, die das Gesetz als juristische Personen betrachtet. Demnach machen sich zwei Arbeiter strafbar, wenn sie sich verabreden, das Großunternehmen, das ein Verbrechen plant, ist hingegen nicht wegen conspiracy strafbar.

73 Markus Dubber, Einführung in das US amerikanische Strafrecht, S. 128, der vor allem auf den US Model Penal Code abstellt. 
von Personen zu schützen und ihre schlimmen Pläne zu vereiteln, als gegen einzelne. "74

Die Absprache mehrerer, ein Verbrechen zu begehen, ist daher deshalb strafwürdig, weil durch die Gruppendynamik, durch das Gefühl der Verpflichtung, durch den Einflussverlust des Einzelnen eine höhere Gefahr für die geschützten Interessen eintritt, als wenn eine Einzelperson einen verbrecherischen Plan fasst. Durch die Verabredung ist der Plan bereits in die Außenwelt gedrungen und spukt nicht mehr nur als „freier“ und daher nicht strafbarer Gedanke im Kopf eines Einzelmenschen umher. Generalpräventiv gewendet ist das Normvertrauen der Bevölkerung bereits erschüttert, sobald der Plan, ein Verbrechen zu begehen, mit anderen gemeinschaftlich gefasst wird.

Stellt man auf diesen Strafgrund ab, so ist die Frage erlaubt, ob es in kontinentaleuropäischen Rechtsordnungen nicht alternative Modelle gibt, dieses Ziel zu erreichen. Darauf weist Jackson explizit hin, wenn er in Bezug auf die Verschwörung zu bedenken gibt, dass es nicht auf das nationale Recht einzelner Staaten ankommt, sondern auf Sinn und Zweck der Regelung der Verschwörung:

„Es erschien wünschenswert, daß den konkreten Fällen die allgemeinen Erwägungen zugrundegelegt werden, wie sie in der Natur des von mir aufgezeigten Problems liegen, als daß sie von den Verfeinerungen irgendeines örtlichen Rechtes beherrscht würden." 75

In der Tat gibt es verschiedene Modelle in unterschiedlichen Rechtsordnungen mit dem gleichen Ziel. ${ }^{76}$

Im deutschen Strafrecht etwa werden zwei Ansätze verfolgt, um der Gefährlichkeit durch Verabredungen mehrerer Personen vorzubeugen bzw. wie Dreher formulierte, die „kriminelle Verteidigungslinie“ nach vorne zu verlegen. ${ }^{77}$ (1) Einerseits wird nach $\S 30$ Abs. 2 StGB (Verbrechensverabredung) der Zeitpunkt des Beginns der Strafbarkeit erheblich vorverlagert. ${ }^{78}$ Die Vorschrift steht allerdings historisch in keinem guten Licht: Zwar war die versuchte Anstiftung bzw. das sich als Täter oder Teilnehmer an einem Verbrechen Anbieten bereits in $\S 49$ a RStGB seit dem 26.2.1876 unter Strafe

74 IMT Bd. 8, 400.

75 IMT Bd. 8, 401.

76 So auch ICTR TC Prosecutor v. Musema, ICTR-96-13, 27.01.2000, §§ 185 ff. mit einem etwas einseitigen Hinweis auf französisches Recht; vgl. auch die Ausführungen von Justice Jackson, IMT Bd. 8, S. $398 \mathrm{ff}$.

77 Eduard Dreher, Grundsätze und Probleme des $§ 49$ a StGB, GA 1954, 11, 12.

78 Die dogmatische Begründung ist indes strittig. Zwar sah die hM in der Vorschrift schon immer eine besondere Form der Vorverlagerung der Strafbarkeit, vgl. LK ${ }^{8}$-Mezger, § 49 a Anm. 2, zu $\S 30$ etwa BGHSt 40, 75; Nachweise bei Thomas Fischer, StGB, 56. Aufl. 2009, §30 Rn. 2 a; es gab aber auch Stimmen, die in ihr eine selbständige Straftat sahen, wie etwa v. Liszt, Strafrecht, 22. Aufl. 1919, S. 804; entsprechend verorteten auch die Entwürfe zu einem neuen Strafgesetzbuch in der Weimarer Zeit im Besonderen Teil, vgl. Eduard Dreher, Grundsätze und Probleme des $\S 49$ a StGB, GA 1954, 11, 12; Karl von Birkmeyer, Teilnahme am Verbrechen, in Vergleichende Darstellung des deutschen und ausländischen Strafrechts, 1908, S. 1, 78-81. 
gestellt; ${ }^{79}$ die hier interessierende Verbrechensverabredung wurde allerdings durch die Strafrechtsangleichungsverordnung vom 29.5.1943 ${ }^{80}$ eingeführt. ${ }^{81}$ Der bundesrepublikanische Gesetzgeber hat sie indes in vollem Umfange durch das 3. Strafrechtsänderungsgesetz bestätigt. ${ }^{82}$ Vorausgesetzt für eine Verurteilung wegen Verabredung wird eine hinreichende Bestimmtheit der Tat. Allerdings müssen Ort, Zeit und Art der Ausführung (noch) nicht verbindlich feststehen; die Tat muss aber in den wesentlichen Grundzügen konkretisiert sein. Entscheidend ist das „Ob“ der Tatausführung. ${ }^{83}$ Die Verbrechensverabredung ist explizit keine eigene Straftat, sondern eine in den Vorfeldbereich verschobene Strafausdehnungsnorm. ${ }^{84}$ Speziell unter Strafe steht im Übrigen die Vorbereitung eines Angriffskrieges nach $\S 80$ StGB.

(2) Andererseits stellt das StGB die bloße Mitgliedschaft in einer kriminellen (terroristischen) Organisation unter Strafe, $\S \S 129,129 \mathrm{a} / \mathrm{b}$ StGB. ${ }^{85}$ Bis 1968 gab es außerdem $\S 128$ StGB, die Geheimbündelei:

„,(1) Die Teilnahme an einer Verbindung, deren Dasein, Verfassung oder Zweck vor der Staatsregierung geheimgehalten werden sollt, oder in welcher gegen unbekannte Obere Gehorsam oder gegen bekannte Obere unbedingter Gehorsam versprochen wird, ist an den Mitgliedern mit Gefängnis bis zu sechs Monaten, an den Stiftern und Vorstehern der Verbindung mit Gefängnis von einem Monat bis zu einem Jahre zu bestrafen."

In der bereits erwähnten Entscheidung im Fall Krulewitch weist Jackson in einer Fußnote darauf hin, dass auch deutsche Gerichte auf der Grundlage von $\S 128 \mathrm{RStGB}$ die NSDAP als verbrecherische Organisation angesehen hätten. ${ }^{86}$ Seiner Ansicht nach basiert diese Annahme auf den gleichen Prämissen wie die Strafbarkeit wegen conspira-

79 RGB1. 25. Auch diese Vorschrift hat seine eigene Geschichte zu Zeiten des „Kultur-Kampfes": Sie kam ins Gesetz als Reaktion auf das Angebot des Belgiers Duchesne an den Pariser Erzbischof und den Jesuitenprovinzal von Belgien, Fürst Bismarck zu ermorden; die Norm war deshalb lange Zeit unter dem Namen „Duchsene-Paragraph“ bekannt; vgl. LK ${ }^{8}$-Mezger, $\S 49$ a Anm. 1; Eduard Dreher, Grundsätze und Probleme des § 49 a StGB, GA 1954, 11.

80 RGBl. I 339.

81 Der BGH stellte in Bezug auf $\S 49$ a StGB fest, dass sie kein typisches NS-Recht enthielte, auch wenn die Strafbarkeit allein subjektiv am Willen des Täters anknüpft. Zur Begründung wird auf eine Parallele zur Versuchsstrafbarkeit verwiesen. Genau genommen handelte es sich in der Entscheidung aber um eine versuchte Anstiftung und nicht um den 1943 neu hinzugekommenen Verabredungsteil der Vorschrift; vgl. Urteil vom 16.2.1951, BGHSt 1, 59, 61 mit Verweis auf OGHbrZ v. 21.6.1949 - StS 27/49; anders hingegen OLG Hamburg MDR $1947,137$.

82 3. StRÄG v. 4.8.1953 - BGBl. I 735.

83 Vgl. BGH NStZ 2007, 697.

84 Vgl. etwa Thomas Fischer, StGB, 56. Aufl. 2009, § 30 Rn. 2; Schönke/Schröder/Cramer/ Heine, Strafgesetzbuch, 26. Aufl. 2006, § 30 Rn. 2; vgl. auch BGHSt 40, 75.

85 Auf diese Besonderheit des deutschen Strafrechts weist auch Jackson hin, vgl. IMT Bd. 8 S. 399.

86 Justice Jackson concurring opinion im Fall Krulewitch v. United States, 336 U.S. 440, 448 Fn. 7: „On conspiracy principles German courts, on May 30, 1924, adjudged the Nazi Party to be a criminal organization. It also held in 1928 that the Leadership Corps of the Communist Party was a criminal organization and in 1930 entered judgment of criminality against the Union of Red Front Fighters of the Communist Party.“. 
cy nach US-amerikanischem Strafrecht. Den entscheidenden Unterschied verkennt Jackson allerdings. Wer wegen bloßer Mitgliedschaft verurteilt wird, ist nur wegen eines Vergehens verantwortlich und erhält nur eine geringe Strafe. Außerdem gehören zu einer kriminellen Vereinigung mindestens drei Personen, die den Willen der Gesamtheit aufweisen und sich untereinander als Verband fühlen, d.h. ein Mindestmaß an fester Organisation aufweisen, während eine Verschwörung auch von nur zwei Personen begangen werden kann, deren Verbindung nicht auf Dauer angelegt sein muss. ${ }^{87}$

Fassen wir die Ausführungen zusammen, lässt sich festhalten, dass das Verbrechen der Verschwörung vor allem zwei Aspekte aufweist: ${ }^{88}$ (1) Durch die Kriminalisierung im Vorfeld der Tatausführung soll eine effektive Prävention gewährleistet werden und zwar sowohl im negativen Sinne, indem die Ausführung verhindert werden kann, wie auch im positiven Sinne, indem das Vertrauen der Bevölkerung in die Stabilität der Rechtsordnung steigt. (2) Durch die Verschwörung lassen sich die individuellen Mitglieder des Kollektives verknüpfen, selbst dann, wenn der Plan nicht zur Ausführung gelangt oder die Plandurchführung differenziert erfolgt und nicht alle Mitglieder daran beteiligt sind. Die Verschwörung trägt demnach auch einen stark beteiligungsrechtlichen Aspekt. ${ }^{89}$

\section{B. Das moderne Völkerstrafrecht (post Nürnberg)}

Der Angriffskrieg als schwerstes internationales Verbrechen wurde nach Nürnberg durch den Völkermord abgelöst. ${ }^{90}$ Die Verabschiedung der Völkermordkonvention am 9. Dezember 1948 mag eine unmittelbare Reaktion auf die Vernachlässigung des Holocausts im Nürnberger Verfahren und Urteil gewesen sein. ${ }^{91}$ Anknüpfend an das Statut des IMT wurde jedenfalls nach Art. 3 (b) der Völkermordkonvention eine Verschwörung zum Völkermord als strafbar angesehen. Die Statuten der UN Tribunale für das ehemalige Jugoslawien (ICTY), Art. 5 Abs. 3 lit. b) ICTY-St., und für Ruanda (ICTR), Art. 2 Abs. 3 lit. b) ICTR-St., kopieren die Konvention in diesem Punkt. Die Verfahrenskammer am ICTR hat einige Prinzipien zur Auslegung und Anwendung der Verschwörungsstrafbarkeit aufgestellt. Demnach setzt conspricay zunächst (objektiv) ein

$87 \mathrm{Zu}$ den Voraussetzungen der Vereinigung statt aller Fischer, StGB § 129 Rn. 6 f.; zuletzt auch hinsichtlich § 129 a StGB BGH NStZ 2008, 146 zum Weltwirtschaftsgipfel, m. Anm. Jahn JuS 2008, 274.

88 Ähnlich Werle, Völkerstrafrecht, Rn. 424: „Die Verschwörung steht dogmatisch zwischen Beteiligungslehre und strafbarer Vorbereitungshandlung.“.

89 Der französischer Richter, Donnedieu de Vabres, Le proès de Nuremberg devant les principes modernes du droit pénal international, in $70 \mathrm{HR}$ (1947-I), 528, 541, stellt vor allem auf diesen kollektiven Aspekt ab und vergleicht die conspiracy mit der französischen complicité.

90 Lüders, Die Strafbarkeit von Völkermord nach dem Römischen Statut für den IStGH, 2004, S. 263; Cryer/Friman/Robinson/Wilmshurst, An introduction to international criminal law and procedure, 2007, p. 165; Vest, Gerechtigkeit für Humanitätsverbrechen?, 2006, S. 143; ebenso ICTR Prosecutor v. Kambanda, ICTR-97-23-S, 4.9.1998, para. 16; Prosecutor v. Serashugo, ICTR-98-39-S, 2.2.1999, para. 15.

91 Vgl. Jost Dülfer, The United Nations and the Origins of the Genocide Convention 1946 1948, in E. Conze/C. Safferling (Hg.), The Genocide Convention, 2009 (im Erscheinen). 
,agreement between two or more persons“ bezogen auf Völkermord voraus. ${ }^{92}$ In subjektiver Hinsicht werden die Anforderungen präzisiert:

„The requisite intent for the crime of conspiracy to commit genocide is ipso facto the intent required for the crime of genocide, that is the dolus specialis of genocide. "93

Das Vorsatzerfordernis bestimmt sich demnach nach dem zugrunde liegenden Delikt, d.h. dem Völkermord. Die subjektiven Anforderungen sind somit denkbar hoch und mit all den Schwierigkeiten behaftet, die bei der Völkermordabsicht im Vollendungsfalle erheblich sind.$^{94}$ Die Strafbarkeit konstituiert sich, ohne dass der Völkermord ausgeführt wird..$^{95} \mathrm{Da}$ in dem konkreten Fall dem Angeklagten Musema allerdings Täterschaft hinsichtlich des Völkermordes nachgewiesen werden konnte, entfällt ohnedies die kriminalpolitische Notwendigkeit, wegen einer zeitlich vorgelagerten Verschwörung zum Völkermord zu verurteilen. ${ }^{96}$ Die Verschwörung ist in diesem Fall dogmatisch ein Durchgangsstadium, dessen Strafbarkeit hinter der vollendeten Tat zurücktritt. ${ }^{97}$ Die Verfahrenskammer am ICTR argumentierte allerdings eher mit einem methodisch zweifelhaften in dubio pro reo-Ansatz und führte aus, dass die für den Angeklagten jeweils günstigste Gesetzesauslegung Anwendung finden müsste. ${ }^{98}$ Das ist in dieser Allgemeinheit sicher nicht haltbar. ${ }^{99}$

Das Römische Statut hat die Kategorie der Verbrechensverschwörung nicht aufgenommen. ${ }^{100}$ Das moderne Völkerstrafrecht kennt keine „Verschwörung“, weder als allgemeine Erscheinungsform des Verbrechens noch gar als eigenständigen Verbrechenstatbestand. Gleichwohl besteht eine Notwendigkeit, dem kollektiven Charakter internationaler Verbrechen gerecht zu werden, denn diese werden in der Regel von einer Vielzahl von Personen begangen, die eine gemeinsame ,policy“ verfolgen. Dazu gehören neben Militärs, paramilitärische Einheiten, Regierungsmitglieder, Diplomaten, ein Verwaltungsapparat, und auch wirtschaftliche Entscheidungsträger. ${ }^{101}$ Dabei stellt es sich häufig als sehr schwierig heraus, die einzelnen Tatbeiträge konkret zu unter-

92 ICTR TC Musema, a.a.O., § 191.

93 ICTR TC Musema, a.a.O., § 192.

94 Vgl. dazu Christoph Safferling, The Special Intent Requirement in the Crime of Genocide, in E. Conze \& C. Safferling (Hg.), The Genocide Convention, 2009 (im Erscheinen) mit weiteren Nachweisen.

95 Cassese, International Criminal Law, S. 229.

96 Alexander Zahar \& Göran Sluiter, International Criminal Law, 2008, S. 183.

97 So auch ICTR TC Musema, a.a.O., §§ 196 ff. Die Argumentation und Methodik der Kammer wird in diesem Punkt scharf kritisiert; vgl. etwa Alexander Zahar \& Göran Sluiter, International Criminal Law, 2008, S. 184; anders auch schon William Schabas, Genocide in International Law, 2000, S. 259 ff. Die Fälle ICTR TC Niyitegeka $\S \$ 429,480,502$ und Nahimana $\S \S 1043-55$ wurde im Übrigen entgegengesetzt entschieden. Auch im englischen Recht ist die Rechtsfolge unklar. Es soll vor allem aus prozessualen Gründen keine Anklage wegen der Verschwörung erfolgen, wenn die Tat durchgeführt wurde; vgl. Glanville Williams, General Principles of Criminal Law, S. 684.

98 ICTR TC Musema, a.a.O., § 198.

99 Vgl. auch Alexander Zahar \& Göran Sluiter, International Criminal Law, 2008, S. 184.

100 Der völkergewohnheitsrechtliche Charakter ist deshalb fraglich; vgl. Cassese, International Criminal Law, S. 228; anders Werle, Völkerstrafrecht, Rn. 425, der vom völkergewohnheitsrechtlichen Charakter ausgeht.

101 Vgl. im „Nürnberger Urteil“ die Ausführungen zur „Verschwörung“ IMT Bd. 1 S. 226. 
scheiden und zu benennen. ${ }^{102}$ Anstatt das Heil in einer Vorverlagerung der Strafbarkeit à la conspiracy zu suchen, sieht die moderne Völkerstrafrechtsdogmatik hierin ein Problem der mittäterschaftlichen Begehensweise. Durch den Wandel vom Täter- zum Tatstrafrecht wird der Ansatzpunkt auf das Geschehen der Außenwelt verlegt und der Plan des Täters tritt in den Hintergrund. Hinsichtlich dieses objektiven Geschehens wird nach der Zurechnung zu Individualpersonen gefragt.

\section{ICTY}

Das Jugoslawientribunal hat zur Zurechnung des kriminellen Erfolgs ein besonderes Modell entwickelt basierend auf dem ,common plan“ oder „common purpose“. 103 Die sog. Joint Criminal Enterprise (jce) kennt drei Zurechnungsstufen. ${ }^{104}$ Während die beiden ersten Stufen grundsätzlich auf der Kenntnis des jeweiligen Mittäters aufbauen, wird über die 3. Stufe der jce der Erfolg einer Einzeltat auch dann zugerechnet, wenn sich die Tat außerhalb des Tatplans bewegt, solange es im Allgemeinen vorhersehbar war, dass derartige Taten geschehen. ${ }^{105}$ Der Vorsatz des Mittäters muss sich demnach gerade nicht auf die Einzeltat, sondern nur auf die Verabredung, den common purpose, beziehen. Der Strafgrund liegt demnach nicht mehr im schuldhaft herbeigeführten kriminellen Erfolg der Tat, sondern in der Verabredung. Diese Zurechnung entspricht weitgehend den „sweeping principles of conspiracy“, wie sie Robert H. Jackson in Nürnberg aufgezählt hat:

„Drittens: Eine Verantwortlichkeit für Handlungen von Mitverschworenen kann auch gegeben sein, obwohl gerade diese Handlungen nicht beabsichtigt oder vorauszusehen waren, wenn sie nur in Ausführung des gemeinsamen Planes begangen wurden. Ein Mitverschwörer macht nämlich den anderen zu seinem Werkzeug mit der Blankovollmacht, die Ziele der Verschwörung zu erreichen. "106

\section{II. $I S t G H$}

Im Statut des IStGH findet sich eine Vorschrift der Mittäterschaft in Art. 25 Abs. 3 lit. a. IStGHSt, die dem Wortlaut nach $\S 25$ Abs. 2 StGB in Erinnerung bringt (,wer ein Verbrechen gemeinschaftlich mit einem anderen begeht"). Bislang ist noch kein Urteil gefällt, welches hierfür Kriterien anbietet, denn die analoge Wortwahl zum StGB muss noch kein Präjudiz für die Interpretation der Vorschrift entsprechend der deutschen Zurechnungsdogmatik darstellen. Im Gegenteil sollte man meinen, dass die (ständige) Rechtsprechung des ICTY in diesem Punkt eine durchaus gewichtige Autorität bildet.

102 Cassese, International Criminal Law, S. 189;

103 ICTY Prosecutor v. Tadic, Berufungskammer, Urteil vom 15.7.1999 (IT-94-1-A), $\S \S 172 \mathrm{ff}$; bestätigt in: Prosecutor v. Krstic, Berufungskammer, Urteil vom 19.4.2004 (IT-98-33-A), § 237.

104 Verena Hahn, Joint Criminal Enterprise, 2008, 248-304; vgl. dazu auch Christoph Safferling, Lernen von Nürnberg, Rechtsgeschichte 14 (2009), S. 148, $163 \mathrm{f}$.

105 Vgl. Kai Ambos, Internationales Strafrecht, 2. Aufl. 2008, § 7, Rn. 23.

106 IMT Bd. 8, S. $402 \mathrm{ff}$. 
In bislang ergangenen Entscheidungen wird obiter intensiv die deutsche Rechtsdogmatik der Tatherrschaftslehre rezipiert ${ }^{107}$ und die Rechtsprechung des ICTY explizit abgelehnt, ${ }^{108}$ was in der (deutschen) Literatur als Bestätigung des Schuldprinzips dankbar aufgenommen wird. ${ }^{109} \mathrm{Ob}$ diese Ansicht reüssieren kann, wird sich zeigen. Auch der IStGH wird auf den makrokriminellen Kontext bei Fragen der Zurechnungsdogmatik reagieren müssen. Ob die Tatherrschaftslehre hier passende Antworten liefert, scheint mir eher zweifelhaft.

Daneben kennt das IStGHSt eine ganze Reihe an Beteiligungsformen, deren jeweilige Abgrenzungen im Einzelnen umstritten sind. Dazu zählen die „Anordnung“, „Aufforderung“ und „Anstiftung“ (Art. 25 Abs. 3 lit. b IStGHSt), ${ }^{110}$ „Beihilfe“ oder „,sonstige Unterstützung“ (Art. 25 Abs. 3 lit. c IStGHSt) ${ }^{111}$ und der „,sonstige (vorsätzliche) Beitrag“ zu einem Gruppenverbrechen, der wenigstens in Kenntnis des Vorsatzes der Gruppe geleistet werden muss (Art. 25 Abs. 3 lit. d IStGHSt). ${ }^{112}$ Diese sehr spezifischen Teilnahmeformen reflektieren indes die Sonderproblematik der conspiracy bzw. der jce nicht.

Auch hinsichtlich des weiteren Aspekts der conspiracy, der Kriminalisierung des Vorfelds durch zeitliche Vorverlagerung der Strafbarkeit, verhält sich das IStGHSt zurückhaltend. Die Strafbarkeit setzt grundsätzlich erst mit dem Beginn der Versuchs ein (Art. 25 Abs. 3 lit. f. IStGHSt). ${ }^{113}$ Dieser setzt neben dem Tatplan voraus, dass ein „wesentlicher Schritt" zur Ausführung der Tat erfolgt sein muss. ${ }^{114}$

\section{Ausblick}

Das Völkerstrafrecht post Nürnberg hat noch kein einheitliches und überzeugendes Zurechnungskonzept entwickelt, das auf die Anforderungen kollektiver Makrokriminalität angemessen reagiert. Die conspiracy, wie sie im angelsächsischen Rechtsraum vor-

107 IStGH Prosecutor v. Thomas Lubanga-Dyilo, ICC-01/04-01/06, Confirmation of the Charges-Entscheidung v. 29.1.2007, $\S 338-342$. Mit Verweis auf Claus Roxin, Täterschaft und Tatherrschaft, 7. Aufl. 2000, S. 294, 299 sowie auf die Beiträge von Eser und Ambos im Kommentar von Cassese/Gaeta/Jones, Commentary on the Rome Statute of the International Criminal Court, 1999.

108 Die Entscheidung beruft sich auf den deutschen Richter am ICTY/ICTR Wolfgang Schomburg in seinem Sondervotum: Prosecutor v. Gacumbitsi, ICTR-2001-64-A, v. 7. Juli 2001, $\S 16$ Fn. 30; a.a.O. Fn. 418.

109 Ambos, Internationales Strafrecht, § 7, Rn. 24 f.; Werle, Völkerstrafrecht,, Rn. 405; Satzger, Internationales und Europäisches Strafrecht, $\S 14$, Rn. 42; Weigend in Münchener Kommentar, § 2 VStGB, Rn. 17.

110 Vgl. Kai Ambos in Otto Triffterer (Hg.), Commentary on the Rome Statute, 2. Aufl. 2008, $\S 25, \mathrm{Rn} .14 \mathrm{f}$.

111 Pellet in Cassese/Gaeta/Jones, a.a.O., Volume I, S. 788.

112 Ambos in Triffterer, a.a.O., § 25, Rn. $24 \mathrm{f}$.

113 Die Aufstachelung zum Völkermord nach Art. 25 Abs. 3 lit. e IStGHSt stellt ein eigenständiges Verbrechen dar und nicht bloß eine besondere Erscheinungsform des Völkermordes; vgl. Ambos, Internationales Strafrecht, § 7, Rn. 48.

114 Werle, Völkerstrafrecht, Rn. 422. Vgl. ausführlich Safferling, Die Abgrenzung zwischen strafloser Vorbereitung und strafbarem Versuch im deutschen, europäischen und im Völkerstrafrecht, ZStW 118 (2006), S. 682, 707-712. 
herrscht, ist international nicht mehrheitsfähig, da es mit rechtsstaatlichen Kautelen, insbesondere mit dem Schuldgrundsatz, nicht zu vereinbaren ist. Immerhin hat auch das IMT von Nürnberg dieses Konstrukt nicht unbesehen übernommen, sondern sehr zurückhaltend nur auf das schlimmste Verbrechen, den Angriffskrieg, bezogen. Des Weiteren hat es für die Zurechnung die Kenntnis des Täters vom Plan und eine raumzeitliche Nähe zur Ausführung verlangt. Das Modell der jce, wie sie die UN Tribunale entwickelt haben, konnte sich nicht entscheidend durchsetzen. Der IStGH jedenfalls übernimmt es bislang nicht. Die völkerstrafrechtliche Zurechnungslehre muss aber auf die Dynamik, die hinter kollektiver Verbrechensverabredung und systematischer Verbrechensplanung wirkt, reagieren müssen. Bislang bleibt der Satz von Robert H. Jackson gültig:

„Kein Rechtssystem hat bis jetzt einen befriedigenden Weg gewiesen, auf dem eine große Anzahl von gleichartigen Anschuldigungen gegen eine große Anzahl von Angeklagten erledigt werden könnte. "115

115 Justice Jackson, IMT Bd. 8, S. 392, der die in Nürnberg angeklagten 21 Individuen für die prozessuale Belastbarkeitsgrenze hielt. 